\title{
Fourier analysis applied to Infrared Thermography of Fiber Composites used for the Strengthening of Structural Elements
}

by L. Cantini, M. Cucchi, G. Fava, C. Poggi

\author{
Dept. of Structural Engineering (DIS) Politecnico di Milano, P.zza L. da Vinci, 3220133 Milan, Italy, \\ cantini@stru.polimi.it \\ cucchi@stru.polimi.it \\ fava@stru.polimi.it \\ carlo.poggi@polimi.it
}

\begin{abstract}
Fiber reinforced polymers (FRP) composites are an efficient method in the strengthening of civil structures. FRP systems are usually applied by means of a wet lay-up method. National guidelines recognize that Infrared Thermography (IRT) is a possible non-destructive evaluation (NDE) technique for the detection of bonding defects, voids and damages and the characterization of the surface layers without a direct contact between the device and the observed area. Conversely, in the guidelines no clear indications are given about the inspection procedure.
\end{abstract}

\section{Introduction}

Fiber Reinforced Polymer (FRP) composites are widely used for the strengthening and seismic retrofitting of concrete, masonry or steel structures. FRP systems are applied using a wet lay-up method in which the fibers are impregnated on site. In this case, the performance of the system is due not only to the quality of the materials but also to the accuracy of the application since the system may contain air voids or non-uniform distributions of the epoxy resin. The efficiency of this technology is strongly dependent on the correct positioning and bonding of the fibers on the surface. Therefore inspection and monitoring are essential.

A series of regulatory documents and guidelines were issued for the use of FRP for strengthening existing civil constructions. In the European fib [1] it is explained that thermographic NDTs may be successfully applied for bonding evaluation and that defects are located as hot (or cool) spots due to different thermal properties of degraded bonding. The $\mathrm{ACl}$ Committee [2] defines that thermographic tests may indicate signs of progressive delamination in the inspection and assessment of reinforced structures. In the Italian Guideline CNR DT200/2004 [3], thermographic tests are considered effective for the evaluation of substrate deterioration prior to FRP application to ensure proper quality of the support and for the execution of non- destructive tests. All the guidelines recognize that the control of the application may be performed with Infrared Thermography (IRT) among different Non Destructive Evaluation (NDE) techniques. Nonetheless, at present, there are no standard procedures to assess the quality of the applications in civil engineering structures. Infrared thermography represents a valid tool for the detection and measurement of bonding defects or damage in the composite strips and can be used for the definition of possible damage progression. The paper presents a series of results obtained using infrared thermography for applications masonry walls and concrete beams. For the analysis of the thermographic sequence of recorded images an algorithm based on the Fourier frequency analysis was set.

\section{Experimental program}

The masonry-CFRP specimen consisted of a brick wall $\left(25 \times 52 \times 102 \mathrm{~cm}^{3}\right)$ while the concrete-CFRP specimen consisted of a C25/30 concrete block $\left(150 \times 150 \times 600 \mathrm{~mm}^{3}\right)$. CFRP reinforcements were bonded using a thixotropic epoxy resin. Lack of bonding was simulated by the interposition of Teflon foils with different dimensions and geometric shapes. The detection of the defects was performed with 1, 2 and 3 layers of FRPs for both the specimens. Figures 1 and 2 show the preparation of the artificial defects applied on the specimens before the application of the FRP strips. Figure 2 present the layout of the different test conditions, with 1, 2, and 3 FRP layers.

A series of thermographic tests was performed to verify the capabilities of IRT technique to estimate the presence of defects. Each artificial discontinuity was previously measured and the real area of the various defects was compared with the geometry estimated by the obtained thermograms.

\subsection{Short test description: evaluation of the thermographic image}

Active IRT is based on the application of heat to a surface to generate a thermal front into the material. In this case, the heating source was applied for a period from 5 to 15 seconds using an infrared lamp positioned at a distance of about $30-40 \mathrm{~cm}$ from the specimen. The electric power of the lamp was $1.5 \mathrm{~kW}$ and the surface was equal to $0.29 \times 0.14 \mathrm{~m}^{2}$. 


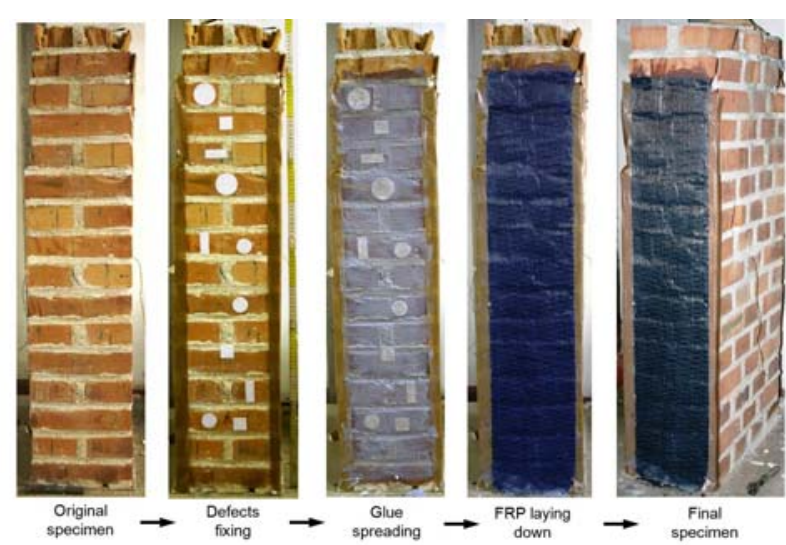

Fig. 1. Preparation of the brickwork specimen.
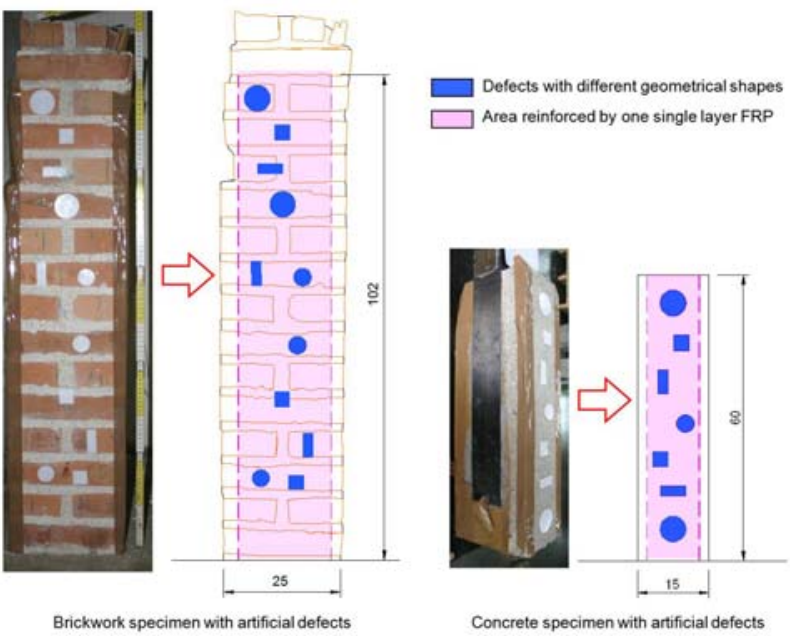

Fig. 3. Layouts of the specimens used for the tests.

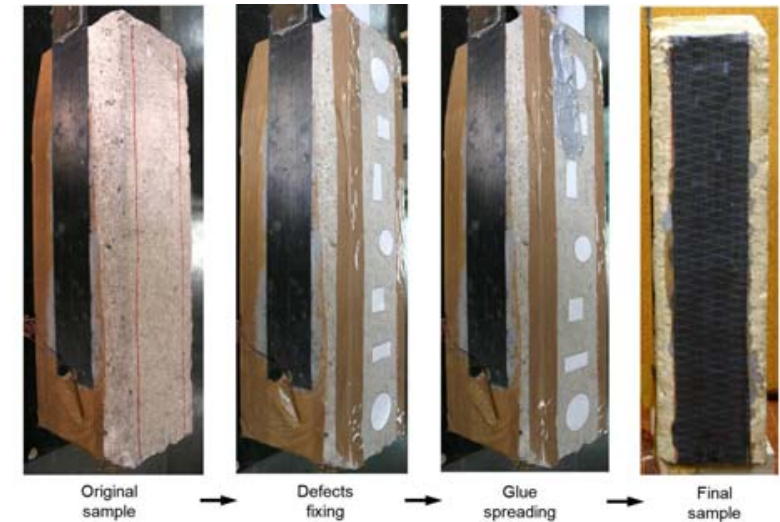

Fig. 2. Preparation of the concrete specimen.

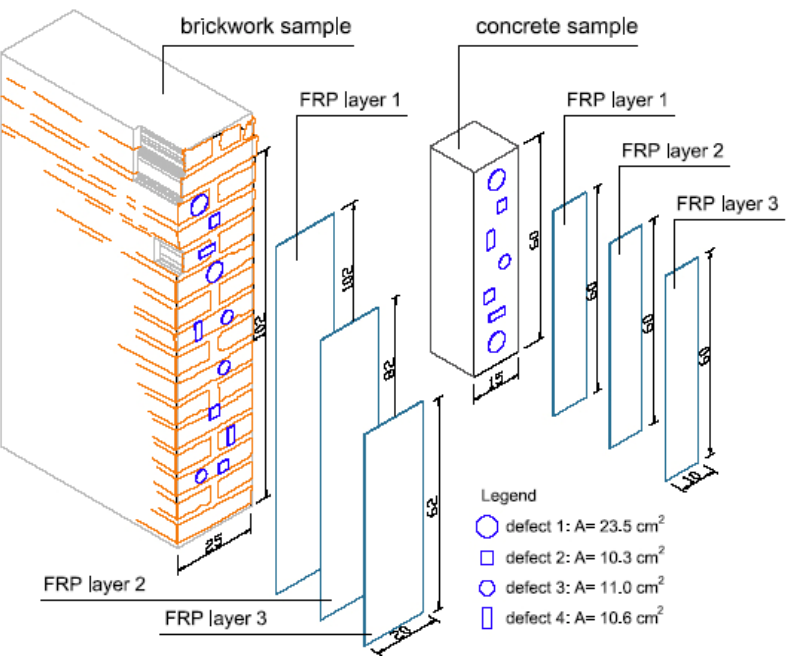

Fig. 4. 3D layout of the specimens and defects geometry.

After external heating, during the cooling phase, the temperature of the specimen surface was recorded in real time by using a thermographic camera positioned at a distance of about $30-40 \mathrm{~cm}$ from the specimen. The IR camera produces images of $320 \times 240$ pixels and is composed of Vanadium Oxide microbolometer detectors allowing to visualize temperatures in a range from $-40^{\circ} \mathrm{C}$ to $500^{\circ} \mathrm{C}$.

The effects of experimental factors and parameters for the analysis and visualization of the bonding defect are therefore taken into account (see figure 5). From an experimental point of view, for the brickwork specimen, an optimal heating time of 5,10 and $15 \mathrm{sec}$ was defined for 1, 2 or 3 FRP layers respectively. For the concrete specimen an ideal time of $5 \mathrm{sec}$ was found irrespective of the number of layers. After the removal of the IR lamp, the cooling time to wait before obtaining the most representative thermogram varies from 3 to $15 \mathrm{sec}$.

After removal of the heating device, the temperature changes can indicate near surface inhomogeneities and the presence of defects in the structure, if they give rise to measurable temperature differences. The results are images recorded during the cooling down phase; showing the distribution of the temperatures through a color scale or a gray scale. These images are also called thermograms and their temperature surface distribution represents the sum of the different discontinuities present behind the external layer. This aspect was observed for the brickwork specimen: the presence of the mortar joints (creating a discontinuity with the bricks) had negative consequences in the defects evaluation. Trying different heating periods, it was observed that the artificial defects could be identified through very fast heating (from 5 to 10 seconds with one FRP strip). Longer exposition to the heating source could warm even the joints with the result of thermograms affected by higher showing a not clear combination of the temperatures conserved by both the mortar joints (due to their porosity) and the artificial defects. Figure 6 presents an example of the typical defects estimation obtained through the thermal cooling evaluation.

In both specimens the defects were identified, for each condition: with one, two and three FRP layers (see figures 7 and 8 ). In each examined condition, the area of the defects was estimated using the available commercial software, able to estimate the dimensions of the framed objects by converting the pixels of the radiometric image into geometric units, if a proportional deferment is known. The data elaboration allowed optimizing the thermal contrast 
between a damaged area and the adjacent zones for the best identification of the position, shape and size of the artificial defects. After heating the specimen, during the cooling phase, the defect estimation presented some differences from the beginning to the end of the test.

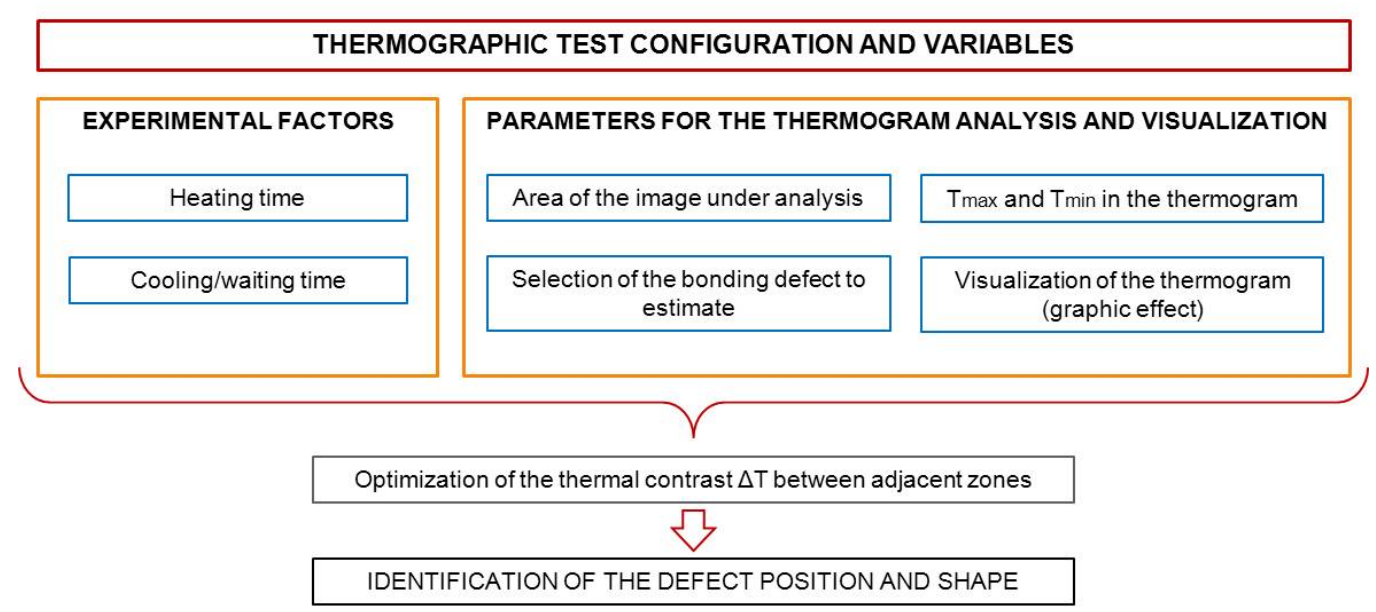

Fig. 5. Thermographic test configuration and variables.
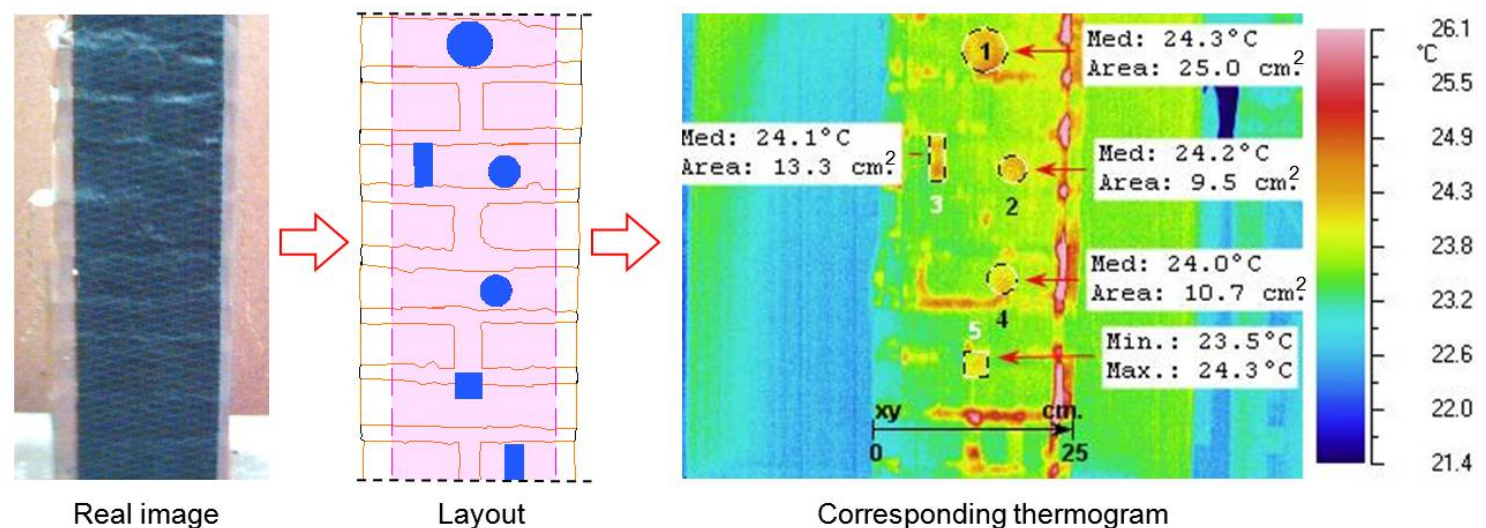

Fig. 6. Example of a defects evaluation obtained through thermal decay observation with the estimation of the defects elaborated by using a commercial software.

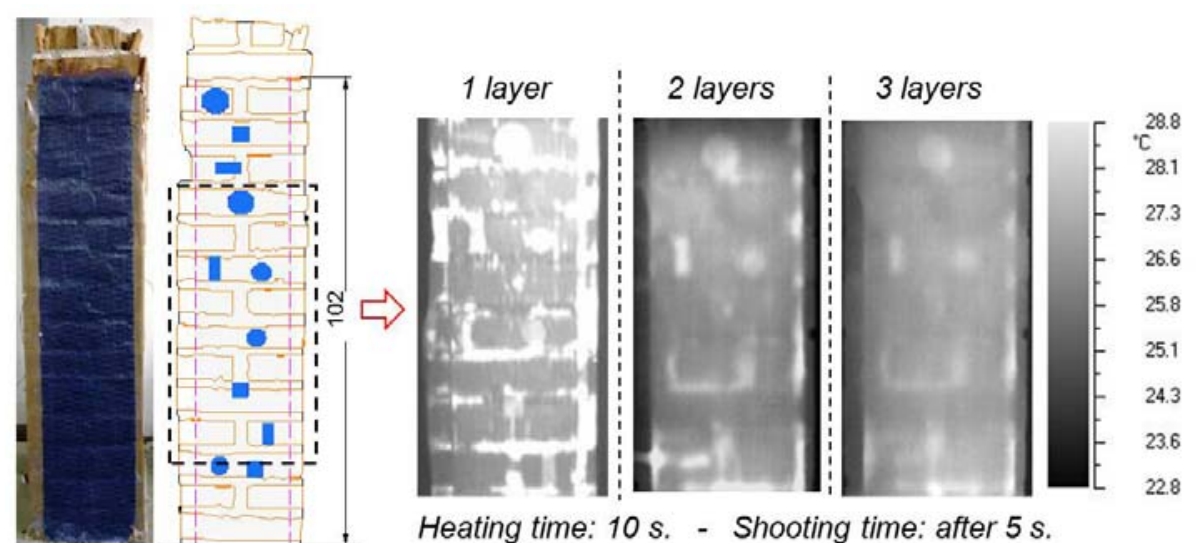

Fig. 7. Comparison between the results obtained on the brickwork specimen through thermal cooling in three different test conditions. 


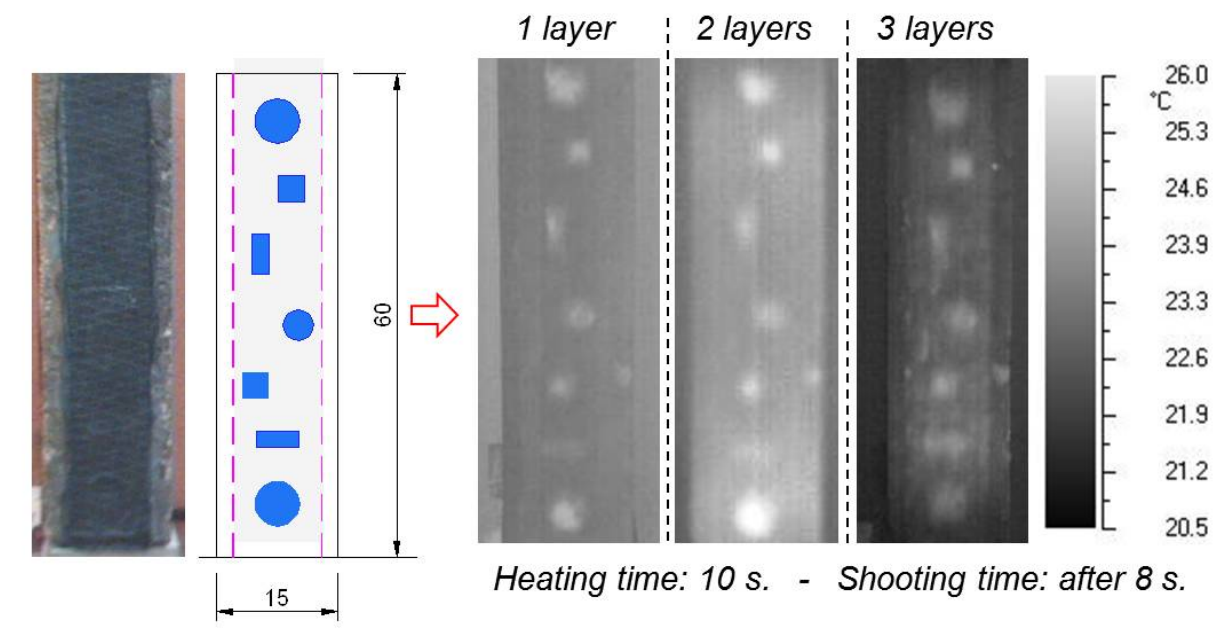

Fig. 8. Comparison between the results obtained on the concrete specimen through thermal cooling in three different test conditions.

For the analysis and visualization of the defect, the thermogram resolution has to be properly adjusted. At first, the area of the thermogram under investigation and the bonding defect to estimate are selected, while the thermal contrast results from the definition of the minimum and maximum temperatures and from the graphic effects the software may provide.

The dimensions of the defects, calculated by the IR camera software, are then based on the accuracy of the operator in recognizing the borders of the defects [4]. This operation is actually time-consuming and influenced by a personal interpretation of the thermal image on the surface of the sample. To improve the research, a procedure based on the Fourier analysis is set and proposed in this paper.

\subsection{Application of pulsed-phase thermography}

Among the various quantitative methods set for thermovision, the Pulse Phase Thermography (PPT), first proposed by Maldague and Marinetti [5-6], can provide interesting results. As shown in the previous example, the traditional pulsed thermographic method are affected by local variation of the emissivity coefficient with negative consequences for the determination of the defects. PPT requires the experimental procedure adopted for Pulse Thermography (PT) and the data elaboration used for another thermographic method based on the modulated heating of the surface, known as Modulated infrared Thermography (MT) or lock-in thermography [5].

Pulsed phase thermography is based on the Fourier transform. As observed before for the pulsed thermography, a sequence of infrared images is recorded in order to analyze the transient cooling following the initial heating. For each pixel of the thermogram, the temporal evolution $f(x)$ is extracted from the image sequence (where $x$ is the index of the image sequence). Recorded surface temperature profiles are then transformed by means of a Fourier Transform algorithm processing the data in magnitude and phase profiles of specific frequencies of the Fourier Transform.

New thermographic tests were carried out on the brickwork specimen. According to Marinetti et al. [7], the quality result of the PPT is defined considering the following parameters:

a) Duration of the observation interval

b) Sampling rate

The signal duration defines the frequency resolution of the phase spectrum. The identification of the defect is a function of the phase difference $\Delta \Phi$ between a reference point and a defect. For this reason, the acquisition duration should contain the interval where defect temperature signal differs from sound zone signal. In practice, this interval is represented by the duration of the thermal contrast. Figure 9, for example, shows the thermal contrast obtained on the brick masonry specimen. The result is referred to an area characterised by three FRP layers applied on 3 artificial defects. The 3 defects are identified in the thermogram, but their geometry is not well defined. In addition, local detachments between the 3 FRP layers provide other negative influences for the correct interpretation of the result.

Considering the $\Delta T$ calculated subtracting the temperature of an area without defects from the temperature measured on a defected area $\left(\Delta T=T_{\text {defect }}-T_{\text {sound }}\right)$, the observation interval was recorded before the appearance of the defect signal and concluded when all the thermal contrast signals became negligible. Figure 10a shows the comparison among two thermal evolutions: one of the sound zone signal and one of the defect zone signal. The corresponding $\Delta T$ is reported in figure 10b. For each observation period used in the thermographic tests, phase different diagrams were obtained. 


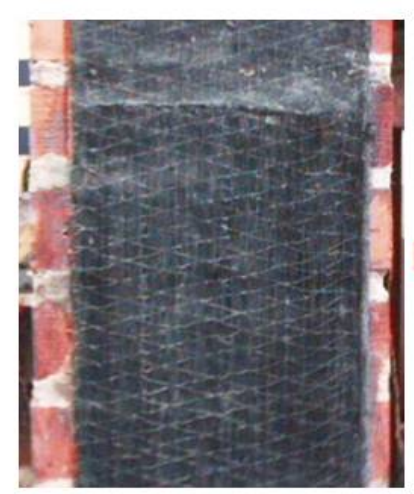

Real image

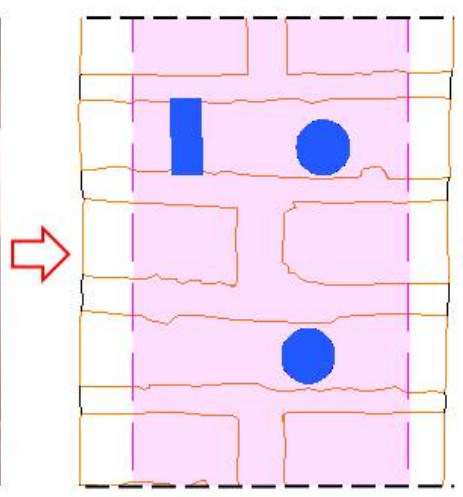

layout

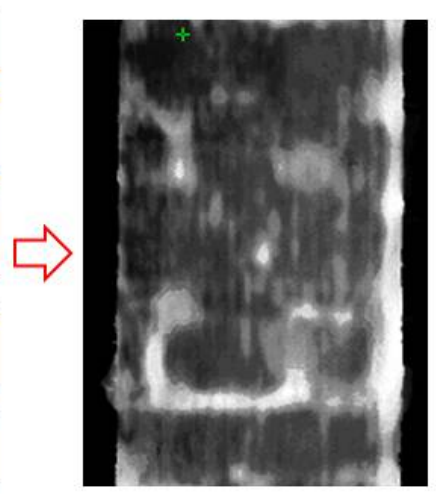

Thermal contrast
Legend

Defects with different geometrical shapes

Area reinforced by three FRP layers

Fig. 9. Thermal contrast result obtained on a detailed area of the brickwork specimen reinforced by 3 FRP layers.

As mentioned above, the acquisition procedure had to be calibrated with a second parameter: the sampling frequency. The sampling rate is connected to the correct determination of the phase difference. It is known that aliasing error distorts the acquired frequency with negative effects on the phase. For this reason three set up configurations, with different sampling frequencies, were used for the further experiments.

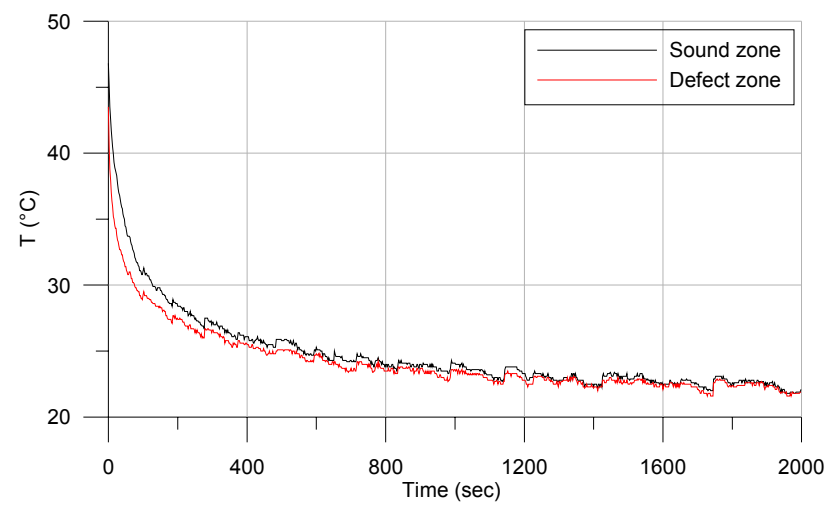

a) Thermal decay curves

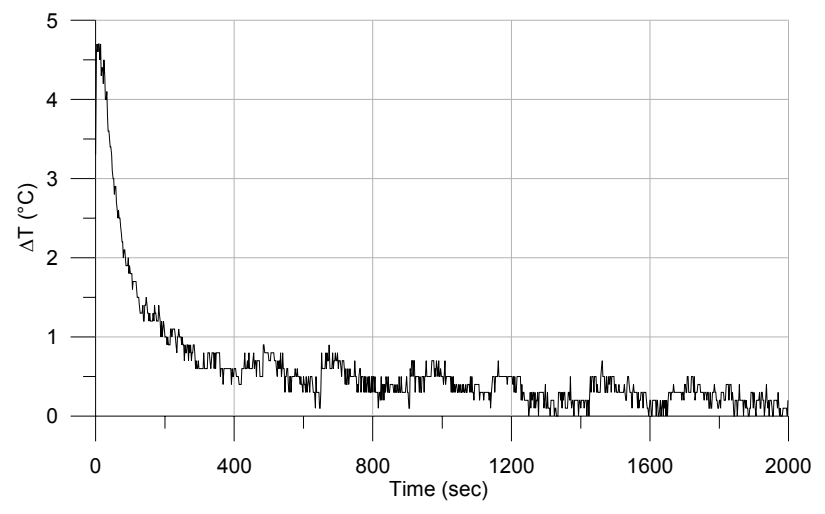

b) $\Delta T$ curve $\left(\Delta T=T_{\text {defect }}-T_{\text {sound }}\right)$

Fig. 10. Temperature evolution curves: a) comparison between sound zone signal and defect zone signal; b) corresponding $\Delta T$ curve.

\section{Results obtained by Pulse Phase Thermography}

PPT tests were carried out on the brickwork specimen, taking into account a specific area characterized by the presence of 3 defects, as shown in Figure 9, covered by 3 FRP layers. According to the theoretical background, the tests were carried out using a duration able to contain the thermal contrast frequencies and a high sample rate. Three different couples of parameters were used for the tests, in order to compare the results of different acquisition modes:

- $\quad$ Case 1: Recording Time $=5000$ seconds; Sampling frequency $=0.2 \mathrm{~Hz}$.

- $\quad$ Case 2: Recording Time $=2000$ seconds; Sampling frequency $=0.5 \mathrm{~Hz}$.

- $\quad$ Case 3: Recording Time $=1000$ seconds; Sampling frequency $=1 \mathrm{~Hz}$.

As shown in Figure 11, each test started after increasing the specimen temperature of about $20^{\circ} \mathrm{C}$ or $30^{\circ} \mathrm{C}$. For each case the recording time was long enough for observing the decreasing of the temperature to the original condition (around $23^{\circ} \mathrm{C}$ ).

Using the Discrete Fourier Transform, magnitude, phase and phase difference profiles as function of frequency were compared in order to evaluate the most reliable acquisition mode. Figure 12 reports the comparison between sound and defect zones for each acquisition set. The three observed cases were used to evaluate the most suitable parameters combination, in order to identify the information for different depths. According to [7], also in the present case, the diagrams reported in Figure $12 \mathrm{c}$ show that the attenuation curve limits the significant frequencies up to $0.01 \mathrm{~Hz}$ : "accurate phase difference measurements are realized if the sampling frequency is greater than the knee in the attenuation curve".

In order to pass from the time domain to the frequency domain, the above described elaboration was carried out for each temperature of the acquired thermograms (composed by $320 \times 240$ pixels). The computation was realized by a home-made software able to read the information contained in the radiometric images acquired through the IR thermo- 
camera. The result is a phasegram, represented by an intensity graph, composed by a matrix of $320 \times 240$ points, where the computed $\Delta \Phi$ corresponds to $z$ axis, at selected frequencies.

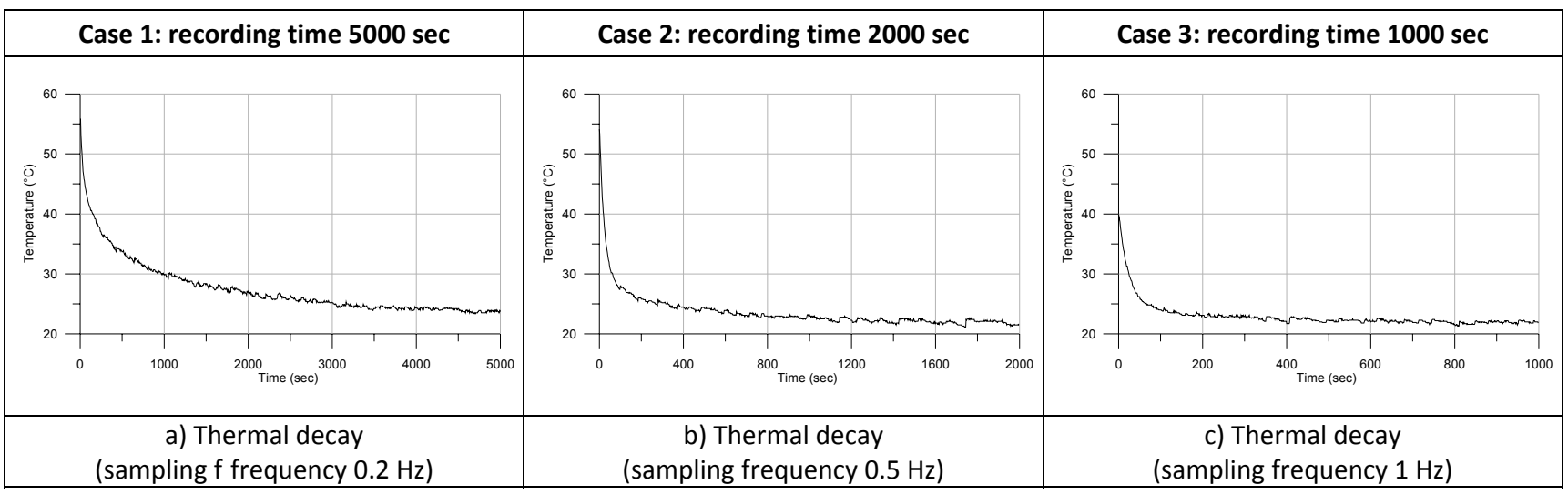

Fig. 11. Comparison between 3 thermal decay curves obtained for each test acquisition mode.

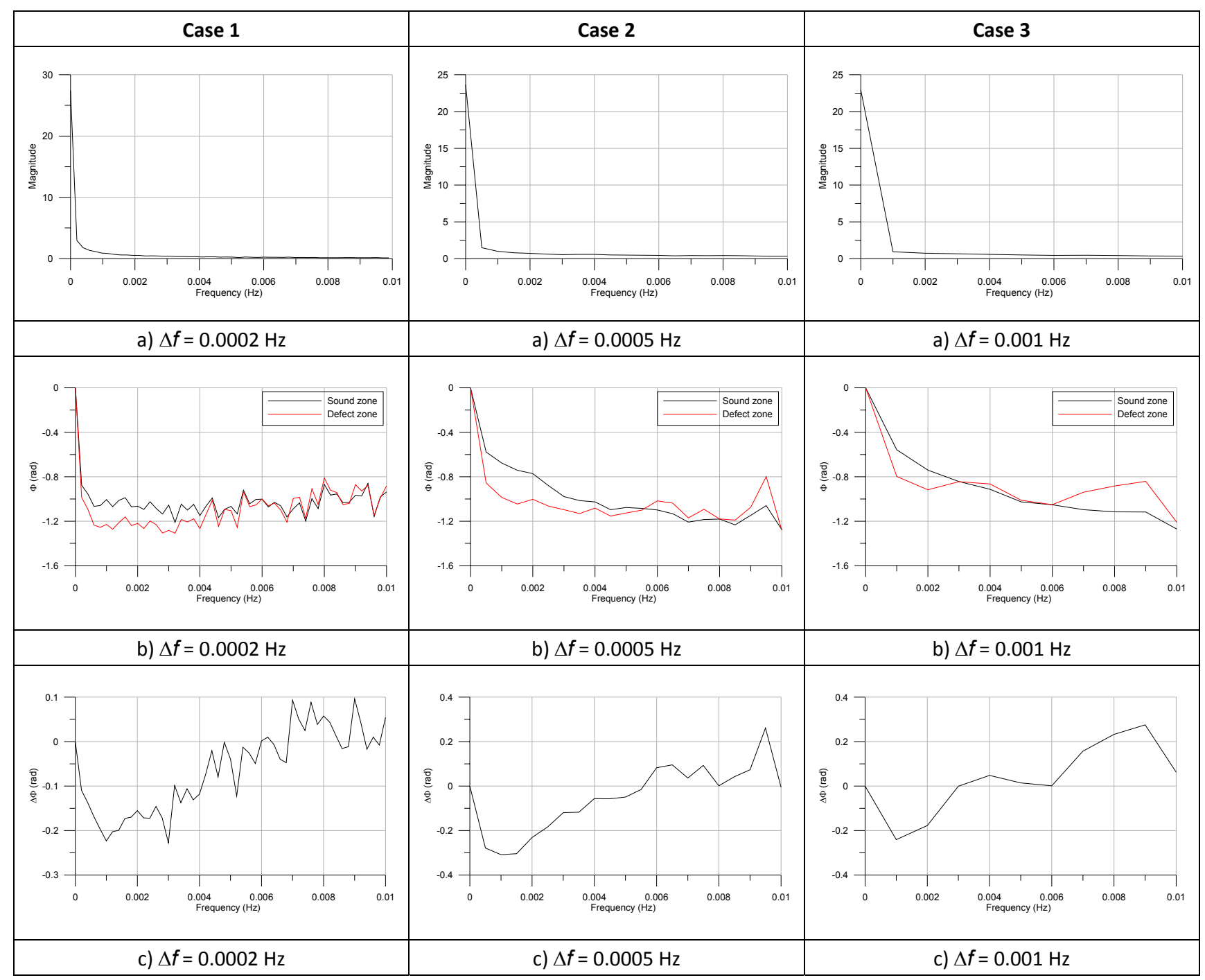

Fig. 12. Magnitude, phase and phase difference profiles as function of frequency on the brickwork specimen for each acquisition mode. 


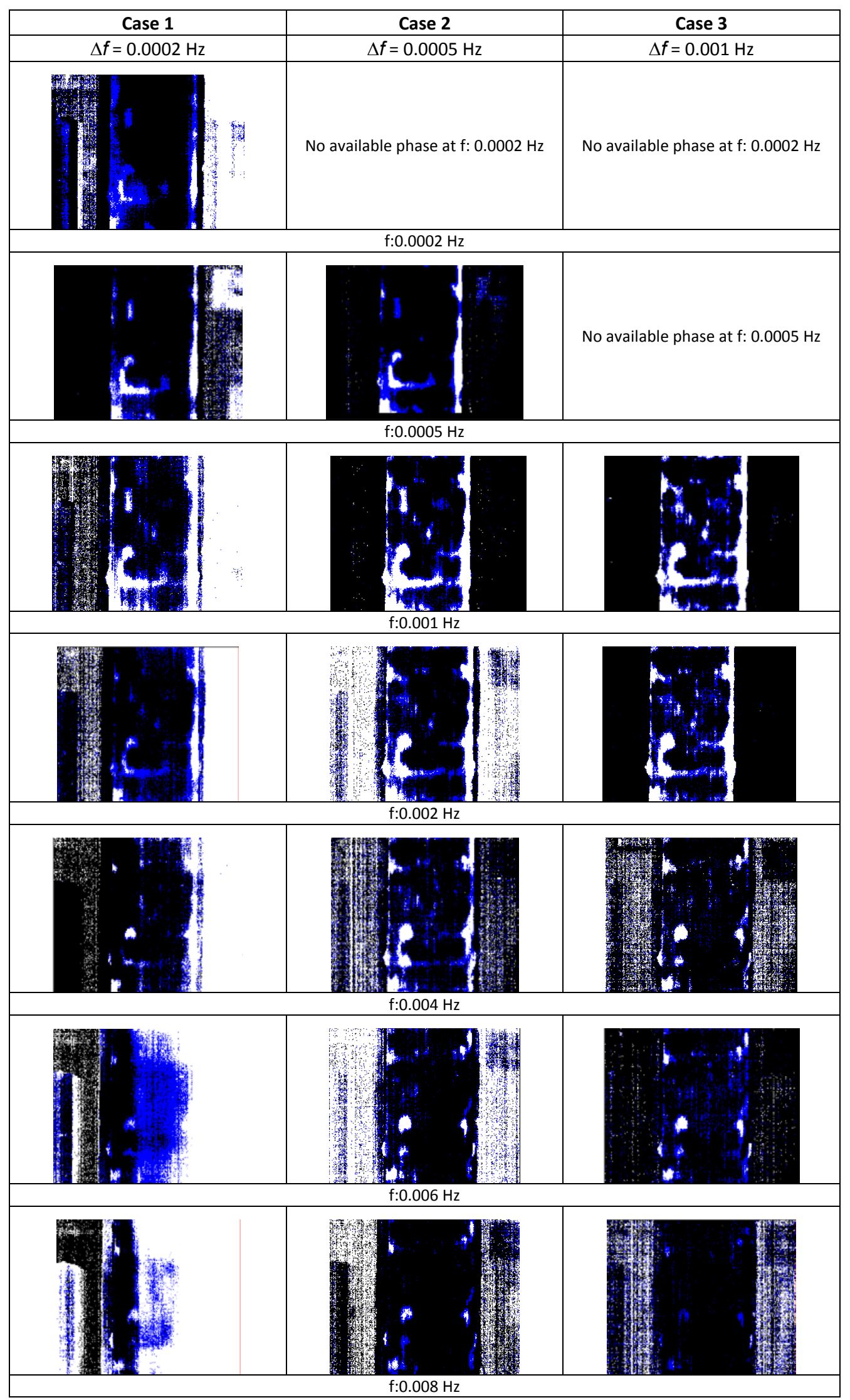

Fig. 13. Phasegrams of the brickwork specimen obtained for each acquisition sets at different frequencies. 
The phase analysis is presented in Figure 13, reporting the phasegrams obtained for the different acquisition sets. The comparison of the different phasegrams enables the evaluation of the debonded areas at different depths. The better quality is obtained in cases 1 and 2, with a recording time of 5000 and 2000 seconds and a resolution that is even too high for the aim of the test is observed. For instance, based on the phasegrams corresponding to $0.001 \mathrm{~Hz}$ (first phasegram for Case 3), the defects at the level of the mortar joint are visible. For higher frequencies only near surface defects (the lack of adhesion between FRP strips) are visible.

\section{Conclusions}

The research focused on the defects evaluation on a brick masonry specimen by thermographic methods. Although the presence of artificial defects simulating the lack of adhesion between the FRP strips and the substrate, mortar joints irregularities and other flaws influenced the interpretation of the results obtained through traditional pulse thermography. On the basis of the operator's experience, the thermal contrast image provided appreciable results: the geometrical shapes of the artificial defects were detected but the quantification of their areas made by a commercial software was not reliable. Furthermore, increasing the FRP layers over the artificial defects, higher differences between the real and the estimated effected areas were observed.

By applying pulse phase thermography, further results showing the defects at different specific depths were obtained. Phasegrams, computed for different frequencies, enable to evaluate the geometrical shapes of the flaws for various levels. These results are useful for distinguishing the defects from the lack of adhesion connected to the mortar joints or the flaws between the FRP layers.

The tests carried out on the masonry specimen were very closed to the typical conditions experimented on site during non-destructive evaluation on reinforced structures. FRP reinforced masonry walls present superficial discontinuities which must be recognised in order to identify the real detached areas. Figure 14 presents the common evaluation of the detachments along a FRP strip applied to a stone wall in a minaret. In this case the distribution of the temperatures is influenced by the irregularities of the masonry walls: the joints effects are indicated.

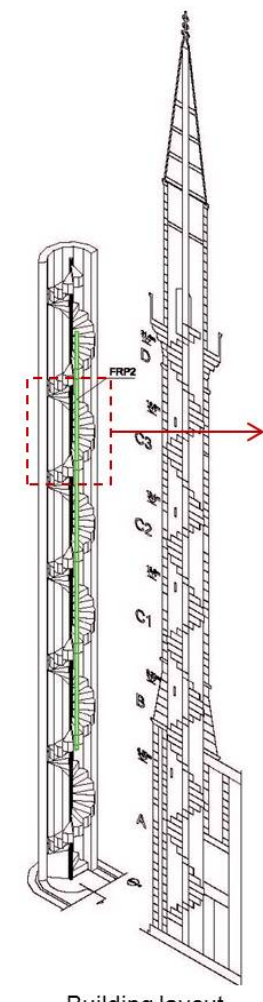

Building layout

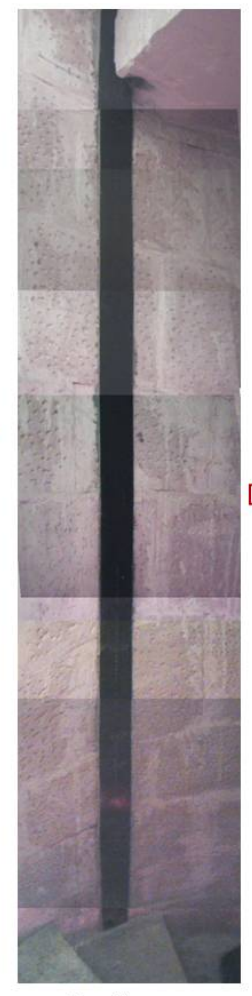

Real image

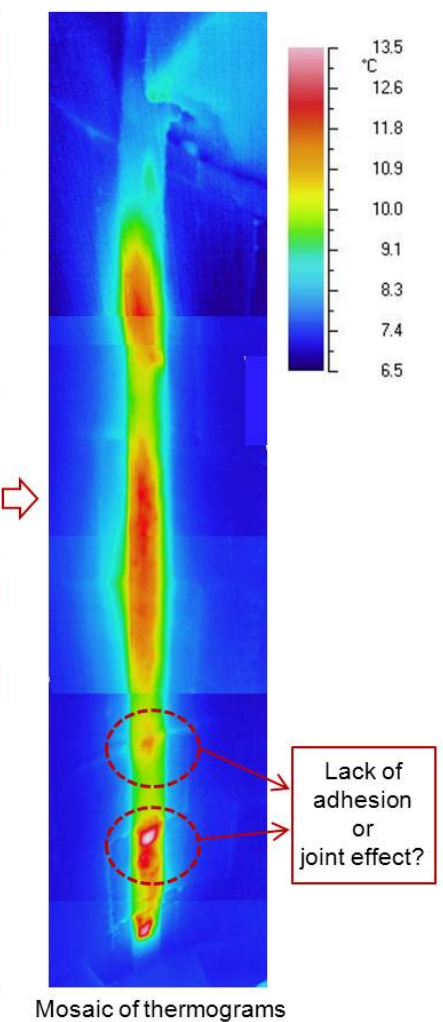

Mosaic of thermograms

Fig. 14. Study of the defects on a FRP strip applied to a stone masonry minaret.

According to the main standards mentioned in the first paragraph, the procedure for evaluating the degraded bonding or alteration of the support is not described in detail. Generic references to thermal contrast image analysis are indicated, but the problems connected to the interpretation of the thermograms are not widely treated. The present work, comparing some laboratory applications, showed the efficacy of the PPT method in discriminating the information obtained at different depth. This result is important for a complete comprehension of the different characteristics of the defects along the interface between FRP strips and the support. Further researches are planned in order to apply the method on real historical buildings presenting FRP reinforcements. Through the new tests campaign, other routines, 
based on the Thermographic Signal Reconstruction (TSR), will be developed with the aim of identifying automatically the shape and the area of each defect in FRP application.

\section{REFERENCES}

[1] fib, (2001). "Externally bonded FRP reinforcement for RC structures", Bull. No 14, Technical Report prepared by Task Group 9.3 Lausanne.

[2] ACl Committee 440. (2002), ACl 440.2R-02-Guide for the design and construction of externally bonded FRP systems for strengthening concrete structures, Farmington Hills, MI.

[3] CNR (National Research Council). (2004), Guide for the Design and Construction of Externally Bonded FRP Systems for Strengthening Existing Structures, Advisory Committee on Technical Recommendations for Construction of National Research Council, Rome, Italy.

[4] Cantini L., Cucchi M., Fava G., Poggi C., "Damage and Defect Detection Through Infrared Thermography of Fiber Composites Applications for Strengthening of Structural Elements", in RILEM Bookseries vol. 6 "Nondestructive Testing of Materials and Structures", Springer, 2012.

[5] Maldague X., Marinetti S., Pulse phase thermography, in Journal of Appl. Physics, vol. 79, pp. 2694-8, 1996.

[6] Maldague X., Galmiche F., Ziadi A., Advances in pulse phase thermography, in Infrared Physics \& Technology, vol. 43, pp. 175-181, 2002.

[7] Marinetti S., Plotnikov Y. A., Winfree W. P., Braggiotti A., "Pulse phase thermography for defect detection and visualization, in Proceedings SPIE (The International Society for Photo-Optical Instrumentation Engineering), Thermosense XXI, April 5-9, 1999, pp. 230-238. 